\title{
Tuberculous retropharyngeal abscess producing respiratory obstruction
}

\author{
N CARROLL, R J I BAIN, M H TSEUNG, R H T EDWARDS \\ From the Department of Medicine, Royal Liverpool Hospital, Liverpool
}

ABSTRACT Oropharyngeal tuberculosis is a rare presentation of the disease even in the presence of extensive pulmonary tuberculosis. A case is described in which a retropharyngeal abscess, causing stridor and threatening respiratory obstruction, was the only manifestation of Mycobacterium tuberculosis infection.

We describe a case of pharyngeal tuberculosis that is unusual both in its presentation-threatened obstruction of the airway-and because there was no sign of pulmonary tuberculosis.

\section{Case report}

A 58 year old merchant seaman, born in Aden and nominally resident in Britain for 30 years, presented with a three day history of increasing difficulty in swallowing and a one day history of difficulty in breathing. He was febrile $\left(37.4^{\circ} \mathrm{C}\right)$, with stridor and an intermittent cough. Bilateral firm, painless cervical lymphadenopathy was present. Direct inspection of the oropharynx showed a large tense mass originating from the posterior pharyngeal wall and almost occluding the airway. Physical examination otherwise showed nothing remarkable; there were no abnormal signs in the chest and no generalised lymphadenopathy or hepatosplenomegaly. The chest radiograph was normal. A lateral radiograph of the neck showed a large soft tissue mass prevertebrally causing almost total occlusion of the airway (figure). There was also extensive cervical osteoarthritis but no evidence of osteomyelitis. Investigations showed normal urea and electrolyte concentrations, a white cell count of 5.8 $10^{9} / 1$ (57\% lymphocytes), a haemoglobin concentration of $12.8 \mathrm{~g} / \mathrm{dl}$ and an erythrocyte sedimentation rate of $57 \mathrm{~mm}$ in one hour.

The mass was incised under local anaesthesia with immediate relief of symptoms. A large amount of pus was removed; microscopic examination showed scanty Gram positive cocci but no acid fast bacilli on Ziehl-Neelsen staining. Bacterial culture was negative. Fine needle aspiration of the cervical nodes showed no evidence of bacterial or mycobacterial infection.

The patient had previously presented in 1986 with a swelling in the neck, which had been surgically explored. An abscess was found and pus that proved sterile was removed. Biopsy of the abscess wall had shown chronic inflammatory

Address for reprint requests: Dr N Carroll, Department of Medicine, University of Liverpool, PO Box 147, Liverpool L69 3BX.

Accepted 30 March 1989 changes only. The patient had been discharged with no follow up.

In view of his present and past medical history he was readmitted two weeks after drainage of the pharyngeal abscess for a cervical lymph node biopsy. Subsequently fluorescent staining of the pus from the original pharyngeal abscess and from the cervical biopsy specimen showed scanty acid fast bacilli, which on culture proved to be mycobacteria with the cultural characteristics of the Asian variety of $M$ tuberculosis. Treatment with Rifinah (rifampicin and isoniazid), pyrazinamide, and pyridoxine was started and he has remained well with no recrudescence of his symptoms or signs during the subsequent six months.

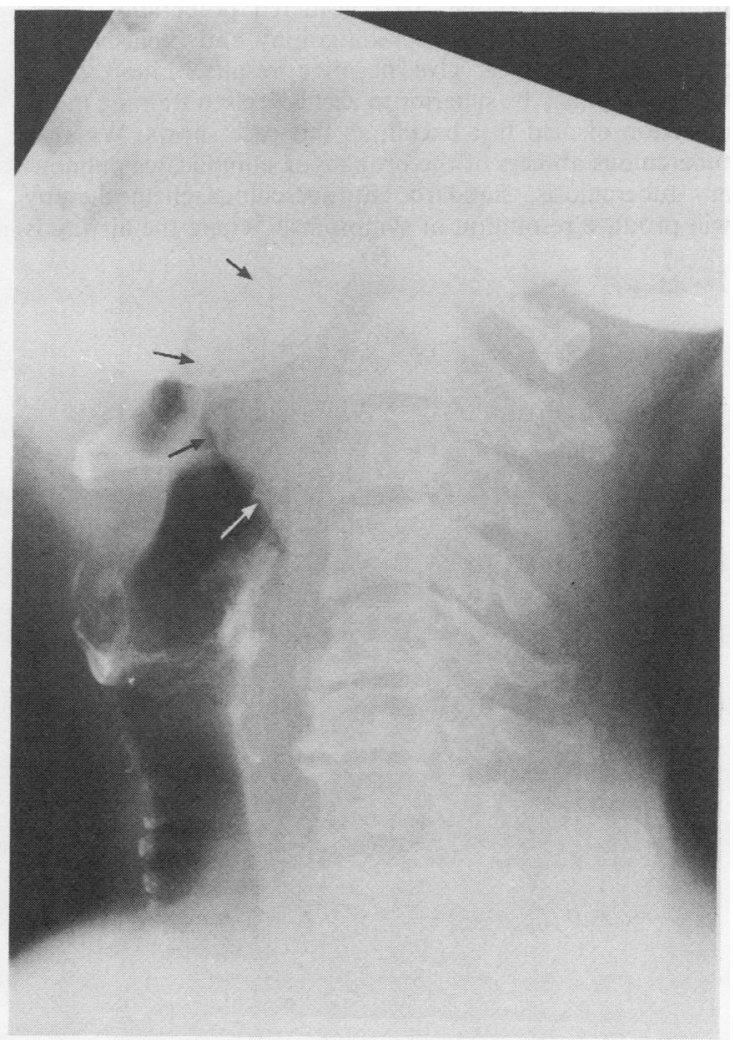

Lateral radiograph of the neck showing a large soft tissue swelling arising prevertebrally in the pharynx and almost occluding the airway. There is extensive cervical osteoarthritis but no evidence of tuberculous osteomyelitis. 


\section{Discussion}

Both primary oropharyngeal tuberculosis and laryngeal tuberculosis are rare..$^{1-3}$ In a review of 20 cases of laryngeal tuberculosis collected over 34 years $80 \%$ had advanced pulmonary tuberculosis. Of 1000 cases of tuberculosis of the upper respiratory tract, $99 \%$ of cases were laryngeal and only $0.9 \%$ had pharyngeal or nasal disease. ${ }^{4}$ In 1950 a case of tuberculous retropharyngeal abscess complicating both pulmonary and cervical node disease was reported. ${ }^{5}$ No dyspnoea or dysphagia was present and, as in the present case, no disease of the cervical vertebrae was noted. The abscess healed after drainage and the use of intramuscular streptomycin. In our patient the previously documented cold cervical abscess was almost certainly tuberculous in nature and was only partially treated by surgical exploration and drainage. At this stage pus was not cultured for $M$ tuberculosis and the histological appearance of the abscess wall was non-specific. The long period before recrudescence may indicate low virulence of this strain of $M$ tuberculosis. At the time the patient presented with stridor there was no clinical, radiological, or bacteriological evidence of tuberculous disease elsewhere.

This case illustrates that all cold abscesses of the oropharyngeal area should be considered potentially tuberculous even if pulmonary radiography and Ziehl-Neelsen staining of the pus give negative results. Fluorescence microscopy may be superior to Ziehl-Neelsen staining in the detection of acid fast bacilli, as this case shows. Where a tuberculous abscess of the orophaynx complicates pulmonary tuberculosis, standard antituberculous chemotherapy will produce resolution of symptoms. ${ }^{6}$ Where the airway is compromised surgical decompression is necessary and direct laryngoscopy is probably best avoided in view of the small $\vec{F}$ but recognised risk of upper airway obstruction. ${ }^{7}$ If a $\stackrel{\mathcal{O}}{+}$ diagnosis of tuberculosis cannot be immediately sustained, biopsy of the area is indicated as laryngeal carcinoma is at least 40 times more common than laryngeal tuberculosis. ${ }^{89}$

\section{References}

1 Sinha SN, Dewan VK. Primary tuberculosis of the larynx. Ear Nose Throat $J$ 1978;57:15-8.

2 Vyravanathan S. Hoarseness in tuberculosis. $J$ Laryngol Otol 1983;97:523-5.

3 Jan A. Primary laryngeal tuberculosis. J Laryngol Otol 1986; 100:605-6.

4 Fernandez-Ponjoan E. Practical considerations from the study of one thousand patients with tuberculosis of the upper respiratory tract. Vida Nueva 1943;51:146-54.

5 Smith MT. Tuberculous retropharyngeal abscess treated by surgery and streptomycin. Arch Otolaryngol 1950;52:767-70.

6 Levenson MJ, Ingerman M, Grimes C, Robbett WF. Laryngeal tuberculosis: Review of twenty cases. Laryngoscope 1984; 94:1094-7.

7 Yarnal JR, Golish JS, Van Derkuyp F. Laryngeal tuberculosis presenting as carcinoma. Arch Otolaryngol 1981;107:503-5.

8 US Department of Health and Human Services. Tuberculosis in the United States, 1978. Atlanta, Georgia: DHHS, 1980. (DHHS Publication No (CDC) 80-8322.)

9 US Department of Health and Human Services. Tuberculosis statistics: states and cities, 1979. Atlanta, Georgia: DHHS, 1980. (DHHS Publication No (CDC) 81-8249.) 